\title{
Spine MRI in Spontaneous Intracranial Hypotension for CSF Leak Detection: Nonsuperiority of Intrathecal Gadolinium to Heavily T2-Weighted Fat-Saturated Sequences
}

\author{
(D)T. Dobrocky, (D) A. Winklehner, (DP.S. Breiding, (D). Grunder, (D). Peschi, (DL. Häni, (DP.J. Mosimann, (DM. Branca, \\ (D). Kaesmacher, (D) P. Mordasini, (D)A. Raabe, (D).T. Ulrich, (D). Beck, (D). Gralla, and (D)E.I. Piechowiak
}

\begin{abstract}
BACKGROUND AND PURPOSE: Spine MR imaging plays a pivotal role in the diagnostic work-up of spontaneous intracranial hypotension. The aim of this study was to compare the diagnostic accuracy of unenhanced spine MR imaging and intrathecal gadoliniumenhanced spine MR imaging for identification and localization of CSF leaks in patients with spontaneous intracranial hypotension.
\end{abstract}

MATERIALS AND METHODS: A retrospective study of patients with spontaneous intracranial hypotension examined from February 2013 to October 2017 was conducted. Their spine MR imaging was reviewed by 3 blinded readers for the presence of epidural CSF using 3 different sequences (T2WI, 3D T2WI fat-saturated, TIWI gadolinium). In patients with leaks, the presumed level of the leak was reported.

RESULTS: In total, 103 patients with spontaneous intracranial hypotension (63/103 [61\%] women; mean age, 50 years) were evaluated. Seventy had a confirmed CSF leak (57/70 [81\%] proved intraoperatively), and 33 showed no epidural CSF on multimodal imaging. Intrathecal gadolinium-enhanced spine MR imaging was nonsuperior to unenhanced spine MR imaging for the detection of epidural $\operatorname{CSF}(P=.24$ and .97). All MR imaging sequences had a low accuracy for leak localization. In all patients, only 1 leakage point was present, albeit multiple suspicious lesions were reported in all sequences (mean, 5.0).

CONCLUSIONS: Intrathecal gadolinium-enhanced spine MR imaging does not improve the diagnostic accuracy for the detection of epidural CSF. Thus, it lacks a rationale to be included in the routine spontaneous intracranial hypotension work-up. Heavily T2weighted images with fat saturation provide high accuracy for the detection of an epidural CSF collection. Low accuracy for leak localization is due to an extensive CSF collection spanning several vertebrae (false localizing sign), lack of temporal resolution, and a multiplicity of suspicious lesions, albeit only a single leakage site is present. Thus, dynamic examination is mandatory before targeted treatment is initiated.

ABBREVIATIONS: CDM = conventional dynamic myelography; DCTM = dynamic CT myelography; FS = fat saturation; Gd = gadolinium; GdM = intrathecal gadolinium-enhanced MR imaging; ICC = intraclass correlation coefficient; PMCT = postmyelography CT; SIH = spontaneous intracranial hypotension

$S_{\mathrm{r}}^{\mathrm{p}}$ pinal imaging has a central role in the diagnostic work-up of patients with spontaneous intracranial hypotension (SIH). It is widely believed that SIH is commonly triggered by CSF leakage from the intrathecal into the epidural compartment (Fig 1). This is due to an osteodiscogenic microspur (calcified disc protrusion or spondylophyte) penetrating the dura or, less frequently, a

Received January 15, 2020; accepted after revision April 6.

From the University Institute of Diagnostic and Interventional Neuroradiology (T.D., A.W., P.S.B., L.G., G.P., P.J.M., J.K., P.M., J.G., E.I.P.), Department of

Interventional, Pediatric, and Diagnostic Radiology (G.P., J.K.), and Neurosurgery (L.H., A.R., C.T.U., J.B.), University of Bern, Inselspital, Bern, Switzerland; Clinical Trials Unit (M.B.), University of Bern, Bern, Switzerland; and Department of Neurosurgery (J.B.), Medical Center, University of Freiburg, Freiburg, Germany.

Please address correspondence to Eike I. Piechowiak, MD, Institute of Diagnostic and Interventional Neuroradiology, Inselspital, University Hospital of Bern, Freiburgstr 8, CH-3010, Bern, Switzerland; email: eike.piechowiak@insel.ch

http://dx.doi.org/10.3174/ajnr.A6592 rupture of a spinal nerve root diverticulum (Fig 2). ${ }^{1}$ In the setting of a ventral dural tear or leaking around a nerve sheath, imaging may show an abnormal accumulation of CSF and aid in localizing the dural defect. ${ }^{2}$ Alternative pathomechanisms without proof of extrathecal CSF collection, like the CSF venous fistula, recently described by Schievink et $\mathrm{al}^{3}{ }^{3}$ may also be demonstrated on imaging.

Several radiologic examinations may be used in the spinal diagnostic work-up of patients with SIH and include unenhanced MR imaging, intrathecal gadolinium (Gd) enhanced MR imaging $(\mathrm{GdM})$, conventional dynamic myelography (CDM), postmyelography CT (PMCT), dynamic CT myelography (DCTM), digital subtraction myelography, and radionuclide cisternography. ${ }^{4-7}$ Some methods are invasive and require intrathecal application of contrast media, and some may be considered complementary. Each examination has its strengths and shortcomings, and different 


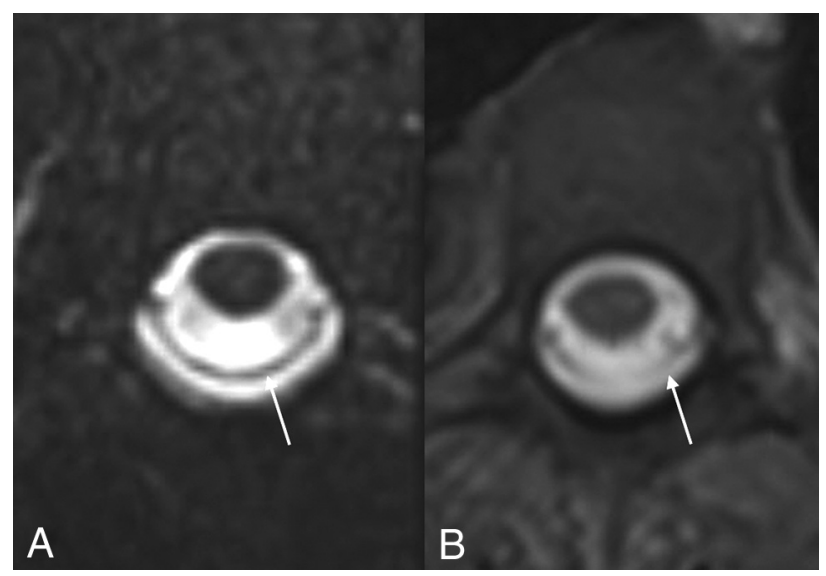

FIG 1. Transversal, isotropic, unenhanced, 3D T2WI FS $(A)$ versus a transversal $(3 \mathrm{~mm})$, T7-weighted sequence with fat saturation after intrathecal gadolinium $(B)$. The extrathecal fluid and contrast media are visible posterior to the dura mater (arrows).

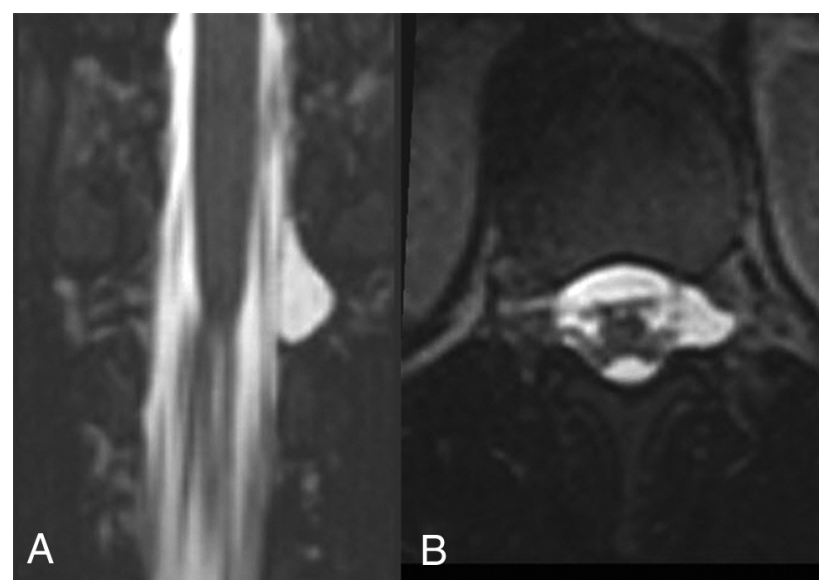

FIG 2. Multiplanar reconstruction of 3D T2WI FS sequence showing a large nerve root diverticulum, which demonstrated a leakage on conventional dynamic myelography (not shown).

methods come into play at different stages of the diagnostic process. Spine MR imaging plays a key role as the first-line diagnostic examination because it offers excellent spatial resolution without associated radiation exposure; however, it lacks any temporal resolution. Previous reports have indicated the diagnostic value of spine MR imaging. ${ }^{8,9}$ Adjunctive GdM was first described in 1999 by Zeng et al. ${ }^{10}$ Since then, several studies have reported its utility in the diagnostic work-up of patients with SIH. Nonetheless, the intrathecal application of Gd has not, so far, been approved by the US Food and Drug Administration and continues to be used off-label.

The goal of our study was to compare the imaging accuracy of 2 different unenhanced spine MR imaging sequences and GdM for visualization of an epidural CSF collection and the accuracy for localizing the precise level of dural dehiscence in the first-line work-up of patients with SIH.

\section{MATERIALS AND METHODS}

This study was granted institutional review board approval, and the need for informed consent was waived due to its retrospective nature. The registry was approved by the local ethics committee (Kantonale Ethikkommission Bern, Switzerland, number: 201700861). Records of all consecutive patients with orthostatic headache and clinical suspicion of SIH evaluated at the University hospital of Bern, Switzerland between February 2013 and October 2017 were retrospectively reviewed. In total, 121 patients with orthostatic headache and clinical suspicion for SIH were investigated according to our routine diagnostic protocol. Patients with postdural puncture headache were not included. Eighteen patients were excluded because of poor-quality MR imaging or absence of MR imaging. The final study population consisted of 103 patients. Patients with a missing spine MR imaging sequence were eligible. In the patients participating in a follow-up study (eg, after microsurgical closure or blood patch), only the preoperative examination was evaluated in our study.

Many patients had been included in previously published studies (study population overlap) investigating different outcome measures, including optic nerve sheath sonography, surgical dural closure, CSF dynamics, DCTM, and brain MR imaging, but none of these studies reported spine MR imaging findings. $1,6,11-14$

\section{Diagnostic Work-Up}

All patients were evaluated according to our standard protocol, as described in previous publications, and are listed below. First, a detailed medical history was obtained, and a physical examination was performed by a neurosurgeon. In most patients, the leading symptom was orthostatic headache; thus, brain MR imaging was performed to rule out any underlying intracranial pathology. The SIH score, based on the 6 most relevant brain imaging findings, which helps predict the likelihood of a CSF leak, was calculated. ${ }^{14}$ Further examinations included optic nerve sheath sonography, ${ }^{12}$ lumbar infusion testing, ${ }^{11}$ unenhanced MR imaging of the spine, GdM, CDM with PMCT, and, in selected patients, DCTM. ${ }^{6}$

\section{Spine MR Imaging}

Unenhanced spine MR imaging was routinely performed in the morning, and GdM, in the afternoon of the same day. Imaging was performed on a $1.5 \mathrm{~T}$ scanner (Aera; Siemens) using a 20-element head/neck coil and a 32-element table spine coil. The unenhanced spine MR imaging protocol included 3 sagittal T2weighted spin-echo blocks $(\mathrm{TR} / \mathrm{TE}=4370 / 93 \mathrm{~ms}$, number of averages $=3$, section thickness $=3 \mathrm{~mm}, \mathrm{FOV}=150 \times 300 \mathrm{~mm}$, FOV phase $=50 \%$, flip angle $=150^{\circ}$, acquisition time $=2$ minutes and 20 seconds, matrix size $=192 \times 512$ ) and 4 sagittal, isotropic 3D heavily T2-weighted turbo spin-echo sequences with fat saturation (FS) (3D T2WI FS) (TR/TE $=1400 / 180 \mathrm{~ms}$, number of averages $=2$, section thickness $=1 \mathrm{~mm}, \mathrm{FOV}=300 \times 300$ $\mathrm{mm}$, FOV phase $=100 \%$, flip angle $150^{\circ}$, acquisition time $=4$ minutes and 33 seconds, matrix size $=640 \times 640$ ) including the entire neurocranium.

Before GdM, written consent for the intrathecal off-label use of a Gd-based contrast agent was obtained from patients. Lumbar puncture was performed with a 22-ga needle with the patient in a lateral decubitus position, and a mixture of $0.5 \mathrm{~mL}$ of Gadovist (gadobutrol, $1.0 \mathrm{mmol} / \mathrm{mL}$, a nonionic macrocyclic agent; Bayer 
Shering Pharma) or $0.5 \mathrm{~mL}$ of Magnograf (gadopentetate dimeglumine, $0.5 \mathrm{mmol} / \mathrm{mL}$; Berlis) with $9.5 \mathrm{~mL}$ of CSF was administered intrathecally.

Three sagittal, isotropic T1-weighted blocks were acquired with the FS technique (T1WI Gd FS) $(\mathrm{TR} / \mathrm{TE}=9.82 / 4.77 \mathrm{~ms}$, number of averages $=1, \mathrm{FOV}=288 \times 288 \mathrm{~mm}$, FOV phase $=$ $100 \%$, flip angle $=10^{\circ}$, acquisition time $=6$ minutes and 9 seconds, matrix size $=288 \times 288$ ). Additional high-resolution, axial T1-weighted blocks with FS were acquired if the neuroradiologist was uncertain whether contrast media was truly present in the epidural space on the basis of the sagittal images $(\mathrm{TR} / \mathrm{TE}=8.62 /$ $4.77 \mathrm{~ms}$, number of averages $=1, \mathrm{FOV}=180 \times 180 \mathrm{~mm}$, FOV phase $=100 \%$, flip angle $=12^{\circ}$, acquisition time $=3$ minutes and 54 seconds, matrix size $=410 \times 512$ ). Alternatively, high-resolution T1-weighted spin-echo blocks with FS in a sagittal orientation were acquired.

\section{Conventional Dynamic Myelography and Postmyelography CT}

The CDM technique, which has previously been described in detail, is summarized briefly below. ${ }^{15}$ For opacification, $20 \mathrm{~mL}$ of Iopamiro 300 (iopamidol; Bracco) was injected intrathecally. Patient positioning was adapted depending on the findings of previous imaging: prone when an osteodiscogenic microspur was suspected or lateral decubitus when a spinal nerve root diverticulum was the presumed source of leakage. The patient was tilted into the Trendelenburg position, following the leading edge of the intrathecal contrast. The level at which the contrast exited the intrathecal compartment and started spilling into the epidural space was considered the level of the dural breach (Fig 3). The patient was immediately transferred to the CT imaging suite, and a PMCT was performed (Somatom Definition Edge; Siemens) to identify possible causative pathology at the level of dural dehiscence or a CSF venous fistula. If no epidural contrast was evident in the first PMCT, a late-phase PMCT was performed, usually 4-24 hours after the initial intrathecal injection, to exclude low-flow leaks.

\section{Data Analysis}

Blinded evaluation of brain MR imaging was performed by a neuroradiology fellow (G.P.), who reported the SIH score. The spine MR imaging of all subjects was assessed independently by a board-certified neuroradiologist (A.W.) and 2 neuroradiology fellows (P.S.B., L.G.), blinded to clinical presentation and to all other imaging studies performed. All spine sequences (T2WI, 3D T2WI FS, T1WI Gd) were separated, deidentified, and sorted randomly on an established, nonmodifiable layout and reviewed on a PACS station. The readers were instructed to perform their assessment and report the results on a standardized spreadsheet, and a short educational module before beginning the image interpretation was provided. For unenhanced spine MR imaging sequences, the presence of CSF in the epidural compartment was recorded; for GdM, epidural contrast accumulation unrelated to the puncture site in the lumbar spine was evaluated. In patients with leaks, the craniocaudal extent was noted (the number of vertebrae with epidural CSF). The readers also reported the number of suspicious lesions potentially causing CSF leakage (such as spondylophyte, disc protrusion, or spinal nerve root diverticula)

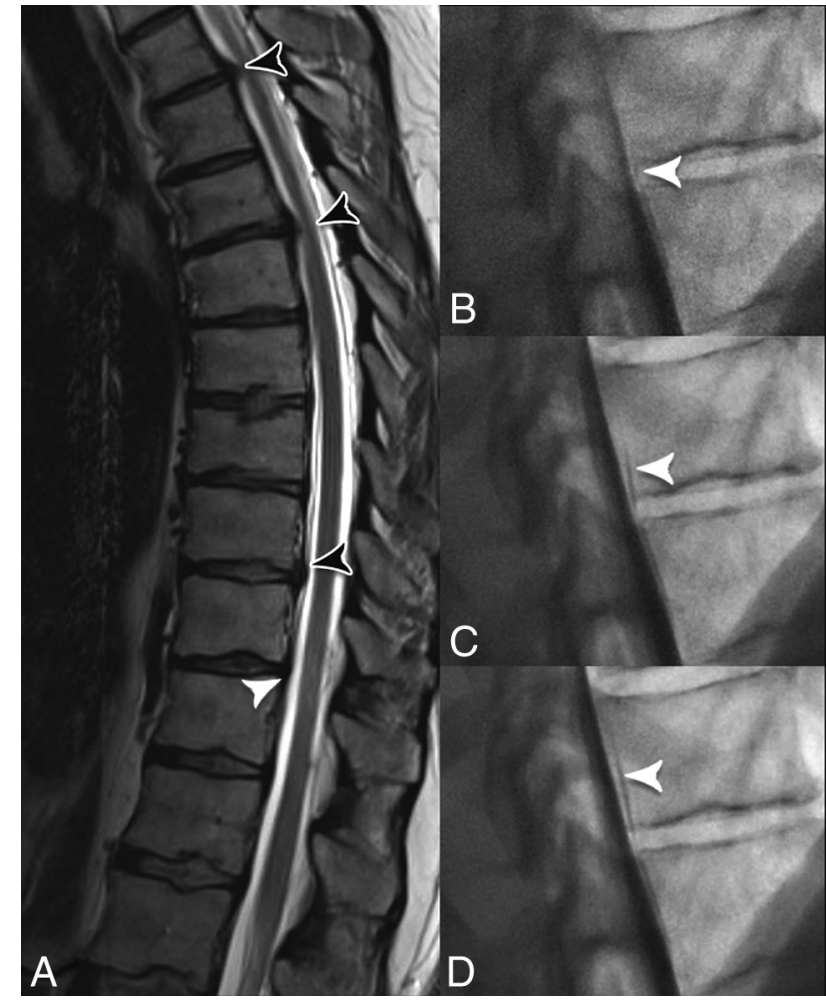

FIG 3. A 57-year-old woman with orthostatic headache and tinnitus. $A$, The sagittal, T2-weighted sequence demonstrates extensive extrathecal CSF and multiple suspicious disc protrusions (black arrowheads). $B-D$, Conventional dynamic myelography demonstrates a CSF leak at the $\mathrm{T10} / 11$ level with progressive contrast media distribution (arrowheads). Even in retrospect, the MR imaging did not demonstrate any suspicious lesion at the corresponding level $(A$, white arrowhead). The leak was confirmed intraoperatively and surgically closed. The follow-up spine MR imaging did not show any residual epidural CSF collection (not shown).

and the lesion they considered the most likely to be the cause of the CSF leak.

In addition, information regarding patients' demographic characteristics (age, sex) and type of therapy (conservative, epidural blood patch, microsurgical closure) was extracted from the registry.

\section{Standard of Reference}

Multimodal imaging including MR imaging, CDM, PMCT, and microsurgical exploration was considered the criterion standard for the proof of a CSF leak. Two neuroradiologists (T.D. and E.I.P. with 8 and 11 years of experience, respectively) who were not involved in the initial analysis reviewed all available images from all patients for the presence of epidural CSF and the level of dural dehiscence. In cases of disagreement, all imaging modalities were reviewed in a joint session to reach a consensus. In patients undergoing microsurgical closure of the CSF leak, intraoperative reports were reviewed to confirm the presence of epidural CSF and verify the localization of the dural breach.

\section{Statistical Analysis}

Statistical analysis was performed using STATA (2017, Release 15; StataCorp). Descriptive analysis was performed using 


\begin{tabular}{lccc}
\hline Sequence available in all patients & $96 / 103(93 \%)$ & $96 / 103(93 \%)$ & $75 / 103(73 \%)$ \\
Sequence available in patients with leaks & $65 / 70(93 \%)$ & $64 / 70(91 \%)$ & $45 / 70(64 \%)$ \\
Craniocaudal extent of the CSF leak & $7.7 \pm 6.2$ & $8.5 \pm 6.9$ & $7.5 \pm 6.7$ \\
$\quad$ (mean No. of vertebrae) & 0.84 & 0.91 & 0.82 \\
$\begin{array}{l}\text { Interrater agreement for the presence of a CSF } \\
\quad \text { leak }\end{array}$ & 0.29 & 0.67 & 0.62 \\
$\begin{array}{l}\text { Interrater agreement for the location of the } \\
\quad \text { CSF leak }\end{array}$ & $4.3 \pm 3.0$ & $5.6 \pm 3.9$ & $5.1 \pm 4.2$ \\
$\begin{array}{l}\text { Mean number of suspicious lesions potentially } \\
\text { causing CSF leakage }\end{array}$ & & & \\
\hline
\end{tabular}

Note:-T2WI indicates T2-weighted; 3D T2WI FS, three-dimensional, isotropic, T2-weighted turbo SE sequence with fat saturation; TWWI Gd FS, isotropic Tl-weighted blocks with fat saturation after intrathecal application of gadolinium. agreement between each sequence and the ground truth using $\kappa$ statistics and bootstrapping methods.

\section{RESULTS}

A total of 103 subjects (63/103 [61\%] women; mean age, $50 \pm 14$ years; range, 25-90 years) with a clinical suspicion of SIH were included. The final study population consisted of $70 \mathrm{SIH}$ leak-positive patients (70/103; 68\%) (48/70 [69\%] women; mean age, 46 \pm 11 years; range, $25-73$ years) and 33 leak-negative patients $(33 / 103$;

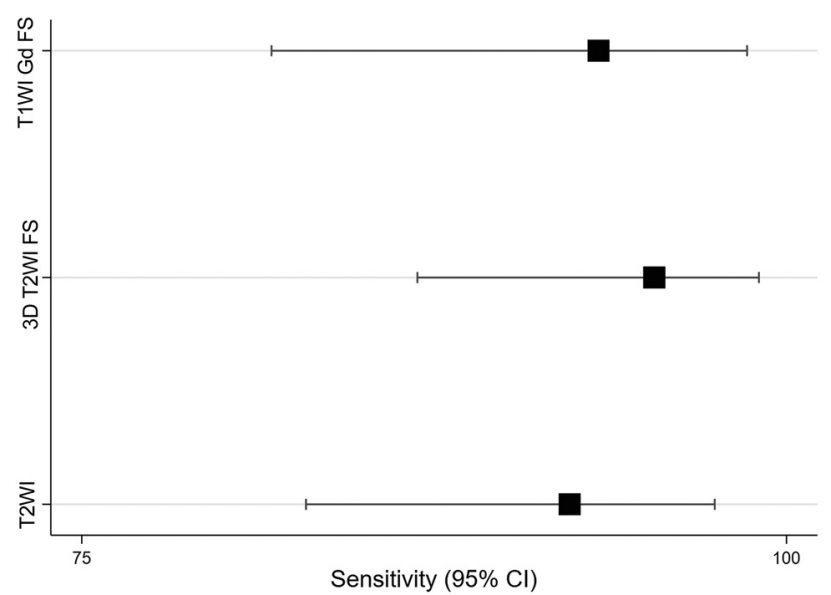

FIG 4. Sensitivity and specificity for the 3 different sequences with corresponding confidence intervals. T2WI indicates T2-weighted; 3D T2WI FS, 3D, isotropic, T2-weighted sequence with fat saturation; and TIWI Gd FS, isotropic, T1-weighted sequence with fat saturation after intrathecal application of gadolinium.

frequencies and percentages for categoric variables and mean $( \pm \mathrm{SD})$ or median (interquartile range) for continuous variables. The results of all 3 readers were aggregated. Continuous measures were averaged among the 3 readers. For dichotomous variables, a qualitative sign was considered positive if 2 or all 3 readers scored it as positive. In cases of categoric variables with $>2$ categories and about which all 3 readers disagreed, the value given by the first reader was the one taken into consideration. The results are presented for the 3 different MR imaging sequences.

Interrater reliability for categoric data was determined using the Fleiss $\kappa$. For continuous data, the intraclass correlation coefficient (ICC) was calculated with the 2-way mixed-effects model estimating absolute agreement. Agreement was defined as almost perfect $(\kappa$ or ICC $>0.80)$, substantial $(\kappa$ or ICC $=0.61-0.80)$, moderate $(\kappa$ or ICC $=0.41-0.60)$, fair $(\kappa$ or ICC $=0.21-0.40)$, or poor $(\kappa$ or ICC $\leq 0.20$ ). If the location was correctly identified, the standard and weighted (with linear weights) $\kappa$ approach was used.

For each MR imaging sequence, scatterplots were used to graphically depict the agreement among the leak location as reported by the readers and the true location. Sensitivity and specificity were obtained comparing the 3 sequences against the ground truth. To test the diagnostic reliability of different sequences for the diagnosis of a CSF leakage, we determined the
$32 \%)(18 / 33$ [55\%] women; mean age, $57 \pm 17$ years; range, $25-$ 90 years) who had orthostatic headache without proof of a CSF leak on multimodal imaging (CDM, PMCT, unenhanced MR imaging, or GdM). The mean brain SIH score was $6.4 \pm 2.2$ in patients with a confirmed spinal CSF leak, and $3.3 \pm 2.5$ in patients without a CSF leak.

All patients ( $n=103,100 \%)$ underwent CDM and PMCT in addition to spine MR imaging. In SIH patients with a leak $(n=70)$, the underlying pathology was a ventral microspur originating from an intervertebral disc or an endplate osteophyte in $48 / 70$ cases $(69 \%)$ and a tear in the nerve root sleeve diverticulum in 13/70 cases (19\%). The remaining 9 patients with an epidural CSF collection showed improvement after conservative management; the precise level of leakage was established on myelography for 7 of them. In 57/70 (81\%) patients with a proved leak on imaging that was refractory to conservative management, microsurgical exploration and dural repair were performed. ${ }^{16}$

\section{Epidural CSF}

Overall, T2WI, 3D T2WI FS, and T1WI Gd FS sequences were available for 96/103 (93\%), 96/103 (93\%), and 75/103 (73\%) patients, respectively (Table 1 ). The craniocaudal extent of the CSF leak (number of vertebrae with epidural CSF) was $7.7 \pm 6.2$, $8.5 \pm 6.9$, and $7.5 \pm 6.7$ for T2WI, 3D T2WI FS, and T1WI Gd FS, respectively. On the basis of the ground truth (multimodal imaging), the presence of CSF in the epidural space was correctly reported in 60/65 (92\%), 61/64 (95\%), and 42/45 (93\%) for T2WI, 3D T2WI FS, and T1WI Gd FS sequences, respectively. The interrater agreement for the presence of a CSF leak was almost perfect for all sequences: T2WI (0.84), 3D T2WI FS (0.91), and T1WI Gd FS (0.82).

3D T2WI FS demonstrated the highest sensitivity (95\%) and specificity (97\%) for the detection of epidural CSF (Fig 4 and Table 2). The $\kappa$ value for T2WI, 3D T2WI FS, and T1WI Gd FS sequences compared with the ground truth was $0.837,0.908$, and 0.833 , respectively. The $\kappa$ statistic comparison between T2WI and 3D T2WI FS ( $P$ value $=.24)$, T2WI and T1WI GD FS $(P$ value $=.97)$, and $3 \mathrm{D}$ T2WI FS and T1WI GD FS $(P$ value $=.25)$ confirmed no relevant differences among the 3 sequences.

\section{CSF Leak Location}

For readers 1,2, and 3, the location of the leak based on $3 \mathrm{D}$ T2WI FS as the most sensitive sequence was identified correctly 
in $27 / 68$ (39.7\%), 24/68 (35.3\%), and 23/68 (33.8\%), respectively. The interrater agreement for the location of the leak was fair for T2WI (0.29), substantial for 3D T2WI FS (0.67), and substantial for T1WI Gd (0.62) (Fig 5).

In 1 patient, an inadvertent epidural injection of Gd occurred and the procedure was successfully repeated on the following day. In another patient, an epileptic seizure shortly after image acquisition occurred. No further adverse reaction that could be attributed to the administration of intrathecal contrast agent was reported.

\section{DISCUSSION}

According to our results, intrathecal Gd-enhanced MR myelography is nonsuperior to unenhanced MR imaging and provides no significant diagnostic benefit in the standard evaluation of spinal
CSF leaks. Additionally, 3D T2WI FS MR images demonstrate a high diagnostic accuracy necessary for the detection of spinal epidural CSF collections. However, our findings clearly demonstrate that unenhanced spine MR imaging and GdM have a low accuracy, which led to poor interrater agreement on localization of the exact level of the spinal CSF leak. This is due to an extensive CSF collection spanning several vertebral levels (false localizing sign); the lack of temporal resolution, which is inherent to the method; and the multiplicity of suspicious lesions that were reported (mean, 5.0); yet in all patients, only a single leakage site was present.

Our results are in line with those of previous studies and support the role of unenhanced spine MR imaging as a valuable, noninvasive tool in the early phase of the diagnostic work-up. ${ }^{8,9}$ We show that isotropic, heavily T2WI with FS is reliable for the detection of a spinal epidural CSF collection, with excellent depiction of anatomy. ${ }^{17}$ Because of similar signal intensities of fluid and fat, standard T2-weighted sequences may mask the epidural CSF collection due to the lack of fat saturation and thus lead to a lower sensitivity (Fig 6). In our experience, multiplanar reconstructions and maximum intensity
Table 2: Sensitivity and specificity or three different MR imaging sequences for detection of epidural CSF $^{\mathrm{a}}$

\begin{tabular}{lccc}
\hline & \multicolumn{1}{c}{ Sensitivity } & Specificity & Youden Index \\
\hline T2WI & $92.3 \%(83.0 \%-97.5 \%)$ & $93.5 \%(78.6 \%-99.2 \%)$ & 0.858 \\
3D T2WI FS & $95.3 \%(86.9 \%-99.0 \%)$ & $96.9 \%(83.8 \%-99.9 \%)$ & 0.922 \\
TIWI Gd FS & $93.3 \%(81.7 \%-98.6 \%)$ & $90.0 \%(73.5 \%-97.9 \%)$ & 0.833 \\
\hline
\end{tabular}

${ }^{a}$ The numbers in parentheses are confidence intervals.

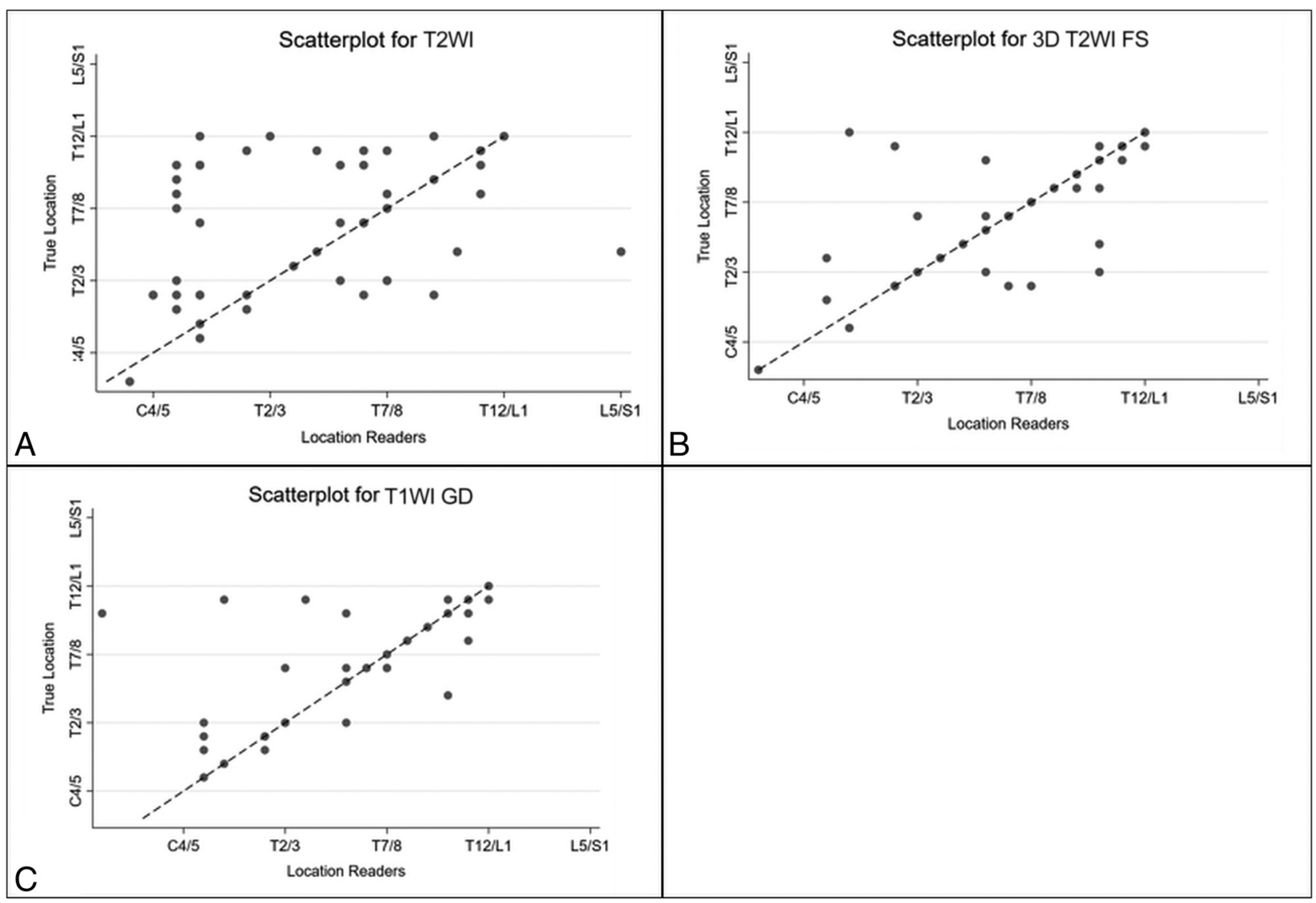

FIG 5. Scatterplots showing the accuracy of each sequence compared with the true location. T2-weighted (A); 3D T2WI FS, 3D T2-weighted sequence with fat saturation (B); and TIWI GD (C). On the $x$-axis is the location as reported by the readers. On the $y$-axis is the true location as found intraoperatively, -57 of 70 (81\%); or as determined with multimodal imaging in conservatively managed patients -13 of 70 (19\%). Patients in whom the leak has been correctly localized based on the corresponding MR image lie on the reference line. 


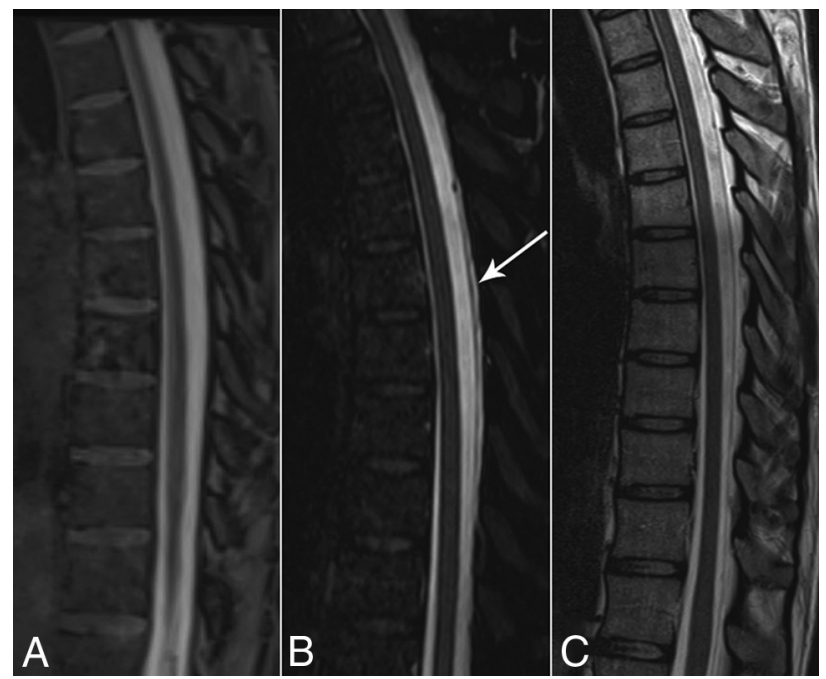

FIG 6. A 38-year-old woman with orthostatic headache and intracranial signs of hypotension (not shown). A, Sagittal, T1-weighted, isotropic sequence after intrathecal gadolinium injection, without proof of epidural contrast media distribution. $B$, Unenhanced, sagittal, heavily T2-weighted isotropic sequence with fat saturation shows a CSF collection in the posterior epidural space and the prominent dural membrane (arrow). C, Due to lack of fat saturation, the epidural CSF collection could not be discerned on the sagittal T2-weighted sequence.

projections are useful for depicting suspicious spinal nerve root diverticula (Fig 2). In addition, unenhanced spine MR imaging is useful to decide on patient positioning for subsequent dynamic myelography: prone when an osteodiscogenic ventral microspur is suspected or lateral decubitus when a ruptured spinal nerve root diverticulum is the presumed source of leakage.

Intrathecal enhanced GdM is an invasive and time-consuming method requiring a lumbar puncture. Its use was first reported in 2005 by Schumacher et $\mathrm{al}^{18}$ in a patient with Marfan Syndrome demonstrating multiple leaks. Chazen et $\mathrm{al}^{5}$ reported a higher rate of CSF leak detection with GdM than with CT myelography and concluded that the method is helpful in the subpopulation of patients with spinal meningeal diverticula. For exact leak localization, in our experience, patients with spinal meningeal diverticula are best evaluated with CDM or DCTM in the lateral decubitus position, which leads to increased contrast concentration along the dependent spinal nerve root diverticulum and may demonstrate contrast leakage or a CSF venous fistula. ${ }^{6}$ Akbar et al ${ }^{19}$ suggested that GdM is a useful adjunctive method for patients with SIH with negative CT myelography findings and demonstrated a leak in $21 \%$ of patients in this subgroup. The increased sensitivity of GdM to slow CSF leaks may be due to repeat myelography rather than a true increase in sensitivity because repeat examinations may reveal intermittent leaks irrespective of the detection method.

Prospective localization of CSF leakage is an endeavor fraught with difficulties. However, it is necessary when microsurgical closure with minimal bone removal or targeted epidural patching is indicated. Epidural CSF accumulation may be misleading because it may not reflect the actual site of the CSF leak (false localizing sign). ${ }^{20,21}$ As reported by Albayram et al, ${ }^{22}$ in 14 of 17 patients with CSF leakage, the site of the dural tear was accurately demonstrated using GdM, which was performed 1 hour after intrathecal Gd administration. Ten of their patients demonstrated a single tear site, and 4 had multiple tears. In our experience, contrast leakage into the epidural space often occurs almost instantaneously, spanning several vertebral levels within a few seconds, making localization on nondynamic imaging impossible and potentially simulating multiple tears. Thus, a GdM scan performed 1 hour after intrathecal Gd is unlikely to show the precise site of leakage in patients with high-flow leaks. In addition, none of our patients with a proven CSF leak $(n=70)$ had multiple leakage sites.

Some hold the misconception that leakage occurs at the level harboring a prominent disc protrusion or a large spinal nerve root cyst, thus obviating dynamic imaging (Fig 3). However, as demonstrated in our study, the mean number of suspicious lesions per patient that could potentially cause a CSF leak was 5 . Consequently, sensitivity for correct localization of the dural leak is poor, with all spinal MR imaging sequences (Fig 5) emphasizing the need for a method with high temporal resolution when targeted therapy is required.

A few case reports have noted adverse effects of intrathecal Gd administration, such as encephalopathy or neurotoxic manifestations, including a decreased level of consciousness, global aphasia, and visual disturbance in humans-some after applying a high dose. ${ }^{23-25}$ In addition, the literature on T1-weighted signal hyperintensities in the globus pallidus and the dentate nucleus in patients receiving serial intravenous administrations of Gd has recently led to a "gado-phobia." ${ }^{26-28}$ Öner et $\mathrm{al}^{29}$ reported similar signal alterations in patients after intrathecal administration of a linear type of Gd. Although an unremarkable clinical follow-up in 51 patients undergoing intrathecal Gd-enhanced cisternography, for an average period of 4 years, was reported by Bakir et $\mathrm{al},{ }^{30}$ the long-term clinical sequelae are not yet certain. Intrathecal Gd remains an off-label use, and the question of whether it provides real added value compared with unenhanced spine MR imaging should be critically reappraised. According to our results, GdM is nonsuperior to unenhanced, heavily T2-weighted spine MR imaging and has been excluded from our standard diagnostic work-up. In fact, it remains a potential field of application in selected cases only (eg, low-flow leaks when all other modalities fail).

The major strength of our study is that leakage and the level of dural dehiscence in a large number of patients have been proved by microsurgical exploration, which is the most precise method. This study has several limitations, the main ones being the retrospective nature of the evaluation, no control group, missing sequences in a small number of patients, and slight differences in MR imaging technique throughout the study because it was not part of a designated study protocol, but a routine MRI.

\section{CONCLUSIONS}

3D T2WI FS is accurate for detection of spinal epidural CSF collection in patients with SIH. GdM is an invasive, off-label method with no added value in the standard diagnostic work-up. All spine MR imaging techniques have a low diagnostic accuracy for the exact localization of the dural breach; thus, a dynamic 
imaging method (eg, CDM) is necessary when microsurgical closure or targeted epidural patching is being considered.

Disclosures: Levin Häni-UNRELATED: Grants/Grants Pending: European Association of Neurosurgical Societies Research Grant, Comments: research grant in neurooncology. Johannes Kaesmacher-UNRELATED: Grants/Grants Pending: Swiss Academy of Medical Sciences/Bangerter Foundation, Swiss Stroke Society*; Travel/ Accommodations/Meeting Expenses Unrelated to Activities Listed: Stryker, Pfizer.* Jan Gralla_UNRELATED: Consultancy: Medtronic/Penumbra, Comments: Global Principal Investigator and clinical events committee member in stroke trials*; Grants/Grants Pending: Swiss National Science Foundation, Comments: stroke studies. * Money paid to the institution.

\section{REFERENCES}

1. Beck J, Ulrich CT, Fung C, et al. Diskogenic microspurs as a major cause of intractable spontaneous intracranial hypotension. Neurology 2016;87:1220-26 CrossRef Medline

2. Schievink WI. Spontaneous spinal cerebrospinal fluid leaks: a review. Neurosurg Focus 2000;9:e8 CrossRef Medline

3. Schievink WI, Moser FG, Maya MM. CSF-venous fistula in spontaneous intracranial hypotension. Neurology 2014;83:472-73 CrossRef Medline

4. Kranz PG, Luetmer PH, Diehn FE, et al. Myelographic techniques for the detection of spinal CSF leaks in spontaneous intracranial hypotension. AJR Am J Roentgenol 2016;206:8-19 CrossRef Medline

5. Chazen JL, Talbott JF, Lantos JE, et al. MR myelography for identification of spinal CSF leak in spontaneous intracranial hypotension. AJNR Am J Neuroradiol 2014;35:2007-12 CrossRef Medline

6. Dobrocky T, Mosimann PJ, Zibold F, et al. Cryptogenic cerebrospinal fluid leaks in spontaneous intracranial hypotension: role of dynamic CT myelography. Radiology 2018;289:766-72 CrossRef Medline

7. Morioka T, Aoki T, Tomoda Y, et al. Cerebrospinal fluid leakage in intracranial hypotension syndrome: usefulness of indirect findings in radionuclide cisternography for detection and treatment monitoring. Clin Nucl Med 2008;33:181-85 CrossRef Medline

8. Starling A, Hernandez F, Hoxworth JM, et al. Sensitivity of MRI of the spine compared with CT myelography in orthostatic headache with CSF leak. Neurology 2013;81:1789-92 CrossRef Medline

9. Watanabe A, Horikoshi T, Uchida M, et al. Diagnostic value of spinal MR imaging in spontaneous intracranial hypotension syndrome. AJNR Am J Neuroradiol 2009;30:147-51 CrossRef Medline

10. Zeng Q, Xiong L, Jinkins JR, et al. Intrathecal gadolinium-enhanced MR myelography and cisternography: a pilot study in human patients. AJR Am J Roentgenol 1999;173:1109-15 CrossRef Medline

11. Beck J, Fung C, Ulrich CT, et al. Cerebrospinal fluid outflow resistance as a diagnostic marker of spontaneous cerebrospinal fluid leakage. J Neurosurg Spine 2017;27:227-34 CrossRef Medline

12. Fichtner J, Ulrich CT, Fung C, et al. Sonography of the optic nerve sheath diameter before and after microsurgical closure of a dural CSF fistula in patients with spontaneous intracranial hypotension: a consecutive cohort study. Cephalalgia 2018;39:306-15 CrossRef Medline

13. Fichtner J, Ulrich CT, Fung C, et al. Management of spontaneous intracranial hypotension: transorbital ultrasound as discriminator. $J$ Neurol Neurosurg Psychiatry 2016;87:650-65 CrossRef Medline

14. Dobrocky T, Grunder L, Breiding PS, et al. Assessing spinal cerebrospinal fluid leaks in spontaneous intracranial hypotension with a scoring system based on brain magnetic resonance imaging findings. JAMA Neurol 2019;76:580-87 CrossRef Medline
15. Kranz PG, Amrhein TJ, Gray L. CSF venous fistulas in spontaneous intracranial hypotension: imaging characteristics on dynamic and CT myelography. Am J Roentgenol 2017;209:1360-66 CrossRef Medline

16. Beck J, Raabe A, Schievink WI, et al. Posterior approach and spinal cord release for $360^{\circ}$ repair of dural defects in spontaneous intracranial hypotension. Neurosurgery 2019;84:E345-51 CrossRef Medline

17. Tomoda Y, Korogi Y, Aoki T, et al. Detection of cerebrospinal fluid leakage: initial experience with three-dimensional fast spin-echo magnetic resonance myelography. Acta Radiol 2008;49:197-203 CrossRef Medline

18. Schumacher $M$, Kraemer N, Berlis A. Intrathecal gadoliniumenhanced MR myelography showing multiple dural leakages in a patient with Marfan syndrome. AJR Am J Roentgenol 2005;185:9294 CrossRef Medline

19. Akbar JJ, Luetmer PH, Schwartz KM, et al. The role of MR myelography with intrathecal gadolinium in localization of spinal CSF leaks in patients with spontaneous intracranial hypotension. AJNR Am J Neuroradiol 2012;33:535-40 CrossRef Medline

20. Hoxworth JM, Patel AC, Bosch EP, et al. Localization of a rapid CSF leak with digital subtraction myelography. AJNR Am J Neuroradiol 2009;30:516-19 CrossRef Medline

21. Schievink WI, Maya MM, Chu RM, et al. False localizing sign of cervico-thoracic CSF leak in spontaneous intracranial hypotension. Neurology 2015;84:2445-48 CrossRef Medline

22. Albayram S, Kilic F, Ozer H, et al. Gadolinium-enhanced MR cisternography to evaluate dural leaks in intracranial hypotension syndrome. AJNR Am J Neuroradiol 2008;29:116-21 CrossRef Medline

23. Reeves C, Galang E, Padalia R, et al. Intrathecal injection of gadobutrol: a tale of caution. J Pain Palliat Care Pharmacother 2017;31:13943 CrossRef Medline

24. Kapoor R, Liu J, Devasenapathy A, et al. Gadolinium encephalopathy after intrathecal gadolinium injection. Pain Physician 2010;13: E321-26 Medline

25. Tali ET, Ercan N, Krumina G, et al. Intrathecal gadolinium (gadopentetate dimeglumine) enhanced magnetic resonance myelography and cisternography: results of a multicenter study. Invest Radiol 2002;37:152-59 CrossRef Medline

26. Radbruch A, Weberling LD, Kieslich PJ, et al. Gadolinium retention in the dentate nucleus and globus pallidus is dependent on the class of contrast agent. Radiology 2015;275:783-91 CrossRef Medline

27. Stojanov DA, Aracki-Trenkic A, Vojinovic S, et al. Increasing signal intensity within the dentate nucleus and globus pallidus on unenhanced T1W magnetic resonance images in patients with relapsing-remitting multiple sclerosis: correlation with cumulative dose of a macrocyclic gadolinium-based contrast age. Eur Radiol 2016; 26:807-15 CrossRef Medline

28. Kelemen P, Alaoui J, Sieron D, et al. T1-weighted grey matter signal intensity alterations after multiple administrations of gadobutrol in patients with multiple sclerosis, referenced to white matter. $S c i$ Rep 2018;8:16844 CrossRef Medline

29. Öner AY, Barutcu B, Aykol Ş, et al. Intrathecal contrast-enhanced magnetic resonance imaging-related brain signal changes: residual gadolinium deposition? Invest Radiol 2017;52:195-97 CrossRef Medline

30. Bakir B, Aydin K, Talu G, et al. Cranial magnetic resonance imaging in spontaneous intracranial hypotension after epidural blood patch. Pain Pract 2008;8:206-10 CrossRef Medline 